\title{
Use of Barbed Sutures for Fixation of Spreader Grafts in Rhinoplasty: A Novel Technique
}

\author{
Allen D Rosen M.D., F.A.C.S.* \\ Assistant Clinical Professor University Hospital Newark, New Jersey, USA \\ Medical Director, The Plastic Surgery Group Montclair, New Jersey, USA \\ Received: December 06, 2017; Published: December 13, 2017 \\ *Corresponding author: Allen D Rosen M.D., F.A.C.S., Assistant Clinical Professor University Hospital, Newark, New Jersey; Medical Director, Plastic Surgery \\ Group P.A., 37 North Fullerton Ave,.Montclair, New Jersey, 07042
}

\section{Abstract}

Barbed suture technology has evolved over the past decade resulting in a variety of new techniques and improved technologies for better fixation of soft tissues throughout the body. The use of barbed suture in facial reconstruction and for cosmetic purposes has been well described. However, the use of this material for fixation of spreader grafts in rhinoplasty has, heretofore, not been reported. This novel technique has improved the efficiency of spreader graft fixation in rhinoplasty, reduces cost and operating time and has a short learning curve.

\section{Introduction}

Dr. Gregory Ruff first developed the concept of placing microscopic barbs along the length of suture in order to provide a more stable fixation for soft tissues in plastic and reconstructive surgery [1]. Angiotech Pharmaceuticals Inc., with the development of the Quill ${ }^{\mathrm{TM}}$ suture retention system for wound closure. In 2007, absorbable barbed sutures became available for commercial use and these have been used in all surgical specialties for soft tissue fixation over the past decade [2]. Covidien ${ }^{\mathrm{TM}}$ and Ethicon ${ }^{\mathrm{TM}}$ have both developed their own barbed suture technologies. These are all variations of a similar theme. They alter a smooth suture with microscopic barbs to allow improved fixation. Quill ${ }^{\mathrm{TM}}$ SRS utilizes bidirectional barbed sutures along the length of a monofilament suture that changes direction at its midpoint. Surgical Specialties, ${ }^{\mathrm{TM}}$ Inc., took over production and distribution of Quill ${ }^{\mathrm{TM}}$, and also offers unidirectional suture products.

This fixates the tissues and eliminates the need for knot tying. The numerous barbs along the length of the suture distribute tension across the wound more evenly than comparable smooth suture products of the same material. In addition, by eliminating the need for knots, the speed at which wound closure can occur is improved, and better scar cosmesis can be achieved. Scar widening and suture extrusion due to the presence of knots along the suture line is eliminated. This timesaving also reduces costs and eliminates the need for additional surgeons for assistance [3]. I have personally been using barbed suture, Quill ${ }^{\mathrm{TM}}$ and STRATAFIX ${ }^{\mathrm{TM}}$, suture since 2007 in both facial aesthetics and body contouring.

Several investigators have reported use of the material in progressive tension sutures for drain less abdominoplasty $[4,5]$ for periareolar breast wound closure [6] and rectus fascia repair [7]. In a report on new and emerging uses of barbed suture I referenced the potential use of barbed suture in rhinoplasty and describe the technique here in greater detail [8].

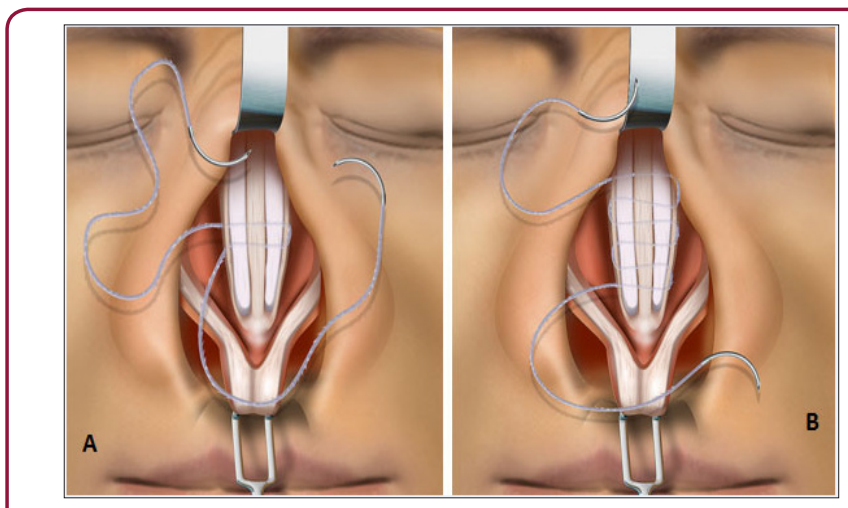

Figure 1: (A) Securing septal cartilage spreader grafts for internal valve repair during rhinoplasty. The first 2 passes with the barbed suture secure and unitize the spreader grafts to the dorsal septum at the transition zone of the bidirectional barbed suture. (B) The spreader grafts are shown completely secured by multiple back-and-forth passes with the bidirectional barbed suture. Reprinted with permission of the manufacturer. (C2013 Angiotech Pharmaceuticals, ${ }^{\mathrm{TM}}$ Inc.

\section{Technique}

The technique for spreader graft placement, preparing the recipient site and harvesting donor cartilage has been described in the literature extensively. Oftentimes, a $3 \mathrm{~mm}$ to $5 \mathrm{~mm}$ wide piece of graft material is utilized and placed on both sides of the dorsal 
septal cartilage. A 3-0 Monoderm Bidirectional Quill ${ }^{\mathrm{TM}}$ suture is placed in the mid-portion of the graft site following placement of the grafts (Figure1A). On one side, the suture is brought through the upper lateral cartilage, spreader graft, dorsal septal cartilage and then out through the spreader graft and upper lateral cartilage on the contralateral side. Gently straightening the curved needle to a more linear, Keith-like, needle is often necessary to facilitate the passage of the suture.

In a serpentine fashion, the suture material is passed from side to side, in the cephalic and caudal, direction from the midpoint fixation where the bidirectional barbs converge (Figure 1B). No portion of the suture material is present along the dorsal contour and additional dorsal contouring is possible following placement of the suture. When completed, the suture is cut at each end without the need for knot tying.

\section{Discussion}

Internal valve collapse in rhinoplasty often requires septal or conchal cartilage for reconstruction by placement of the cartilage graft between the dorsal septum and the upper lateral cartilages. This improves internal valve patency and assists with airflow in patients suffering from internal valve collapse. Following cosmetic rhinoplasty, it also allows for improvement in dorsal aesthetic lines and can be used to straighten a deformed dorsal septum. It can also before provide lengthening of short noses via tongue-ingroove septal extension techniques. The fixation of spreader grafts has often been performed with interrupted horizontal mattress sutures of monofilament, permanent or absorbable materials. It has always been critical to avoid placement of suture material over the dorsal aspect to minimize the palpability or visibility of sutures and to allow additional dorsal contouring at the conclusion of the procedure. The placement of multiple interrupted sutures along the length of the graft is oftentimes made technically more difficult with knot tying in the limited space, even in open rhinoplasty techniques. It is for this reason that I began using absorbable barbed suture for fixation of cartilage spreader grafts for internal valve reconstruction.

\section{Conclusion}

The use of barbed suture for cartilage graft fixation in internal valve reconstruction provides a more secure, unitized fixation with continuous suture distributing tension forces throughout the entire graft site. The process is quick, with an easy learning curve. It reduces the time needed to knot interrupted sutures. In addition, it has eliminated mobility of cartilage grafts and, therefore graft migration or distortion. It has become my exclusive technique for spreader graft fixation in reconstructive and cosmetic rhinoplasty. I believe additional novel uses of barb suture technologies will help evolve additional new techniques to help surgeons reduce operative time, reduce cost, improve soft tissue fixation, and achieve better results.

\section{References}

1. Ruff G (2006) Technique and uses for absorbable barbed sutures. Aesthet Surg J 26(5): 620-628.

2. Zaruby J, Gingras K, Tayor J, Maul D (2011) An in vivo comparison of barbed suture devices and conventional monofilament sutures for cosmetic skin closure: biomechanical wound strength and histology. Aesthetic Surg J 31(2): 232-240.

3. Paul M (2009) Bidirectional barbed sutures for wound closure: evolution and applications. J Am Coll Certif Wound Spec 1: 51-57.

4. Rosen, Allen D (2001) Use of absorbable running barbed suture and progressive tension technique in abdominoplasty: a novel approach. Plast Reconstr Surg 125(3): 1024-1027.

5. Warner JP, Gutowski KA (2009) Abdominoplasty with progressive tension closure using a barbed suture technique. Aesthetic Surg J 29(3): 221-225.

6. Rosen AD (2015) Periareolar Closure with Barbed Suture. Aesthetic surgery journal / the American Society for Aesthetic Plastic surgery 36(3): 372-375.

7. Rosen A, Hartman $T$ (2011) Repair of the midline fascial defect in abdominoplasty with long-acting barbed and smooth absorbable sutures. Aesthet Surg J 31(6): 668-673.

8. Rosen AD (2013) New and Emerging Uses of Barbed Suture Technology in Plastic Surgery. Aesthetic Surgery Journal 33(3suppl): 90S-95S.

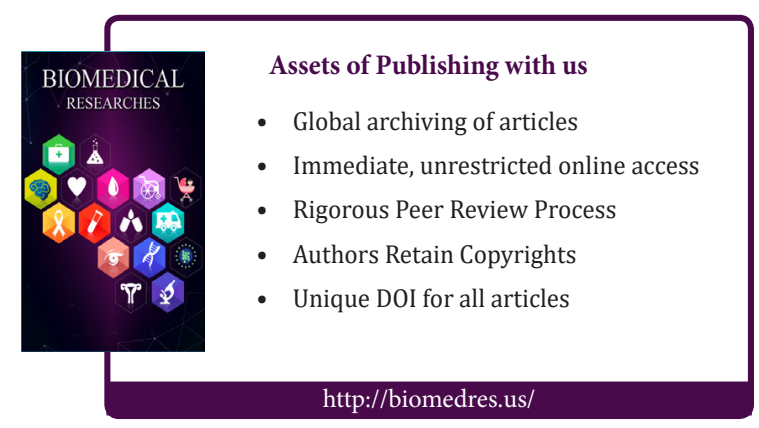

